\title{
Cefalea crónica diaria con Abuso de Analgésicos
}

\author{
Noelia Fontanillas Garmillaa , Julio Pascual Gómez ${ }^{\mathrm{b}}$, Rafael Colás Chacarteguic
}

\begin{abstract}
a Médico de Familia. Centro de Salud de Miera, Cantabria, España.

b Especialista en Neurología, Hospital Universitario Marqués de Valdecilla, Santander, España.

c Médico de Familia.

Centro de Salud de Santoña,

Cantabria, España.

Correspondencia:

Noelia Fontanillas Garmilla,

Centro de Salud de Miera,

C/ Generalísimo s/n, 39722 -

Liérganes, Cantabria, España.

Telf.: 942528556.

Correo electrónico:

noefonta@yahoo.es
\end{abstract}

Recibido el 4 de julio de 2009.

Aceptado para su publicación el 10 de agosto de 2009.

\section{RESUMEN}

La cefalea crónica diaria es una entidad frecuente en la población general. Un alto porcentaje de pacientes con cefalea crónica diaria tiene un consumo abusivo de analgésicos que hace que la cefalea empeore y además puede tener riesgo de múltiples patologías producidas por estos fármacos. El médico de familia ocupa una posición privilegiada para la detección e intervención de esta patología, consiguiendo con ello una mejoría de la cefalea, un aumento de la calidad de vida del paciente y una disminución del gasto sanitario.

Palabras clave: Cefaleas Secundarias. Analgésicos.

\section{ABSTRACT}

Chronic daily headache with analgesic abuse

Chronic daily headache is common in the general population. A high percentage of patients with chronic daily headache overuse analgesics. This contributes to a worsening of the headache and increases the risk of other conditions secondary to analgesic overuse. The family doctor is in a good position to detect and rectify this abuse, resulting in headache relief, increased quality of life and a reduced pharmaceutical cost.

Key words. Headache Disorders, Secondary, Analgesics.

\section{EPIDEMIOLOGÍA}

La cefalea crónica diaria (CCD) es frecuente en la práctica clínica diaria. Dado que la mayoría de los enfermos que consultan por este motivo abusan de analgésicos, este factor se ha puesto directamente en relación con la cronificación de la cefalea primaria de base. España ha sido pionera en el estudio de la epidemiología de la CCD. En población general se estima una prevalencia de CCD del $4,7 \%^{1}$ y una prevalencia del $1,5 \%$ de pacientes con cefalea crónica y abuso de analgésicos (CAA) ${ }^{2}$, por tanto aproximadamente un tercio de los pacientes con CCD cumplen criterios de abuso de analgésicos. En Unidades de cefalea aproximadamente el $40 \%$ de los pacientes tienen $\mathrm{CAA}^{3}$, lo que confirma la elevada prevalencia del abuso de analgésicos por cefalea.

\section{CEFALEA CRÓNICA Y ABUSO DE ANALGÉSICOS}

En los años 50 se realizan las primeras descripciones de pacientes cuyas cefaleas empeoraron al consumir diariamente ergotamina ${ }^{4}$ y posteriormente aparecen nuevos estudios que implican analgésicos simples y opioides en la cronificación de la cefalea, dando lugar al concepto de cefalea por abuso de analgésicos o cefalea de rebote ${ }^{5,6,7}$. En los años 90 se publican los primeros trabajos que implican a los triptanes en el mantenimiento $\mathrm{y} / \mathrm{o}$ agravamiento de la cefalea preexistente ${ }^{8,9}$.

Otros autores han tratado de investigar si el consumo de analgésicos por sí mismo puede producir cefalea crónica en pacientes sin antecedentes de cefalea primaria. Se han realizado estudios en grupos de pacientes que tomaban analgésicos diariamente por dolor músculo-esquelético o procesos reumatológicos, no encontrando mayor prevalencia de cefalea crónica que en la población general ${ }^{10,11}$. 
Estos datos sugieren que los analgésicos por sí mismos no son causa de cefalea crónica en pacientes sin historia previa de cefalea, pero sí pueden empeorar la cefalea episódica contribuyendo a su cronicidad.

La fisiopatología de la CCD y CAA es desconocida, aunque cada vez son más los autores que defienden que el factor etiológico principal sea genético, es decir, estos pacientes nacen ya con una predisposición, genéticamente determinada, para el desarrollo de una cefalea crónica. En algunos casos la predisposición sería tan fuerte que ya desde edades iniciales de la vida y sin otros factores el paciente desarrolla "espontáneamente" una CCD. En el resto de los pacientes, la CCD (y por ende la CAA) aparecería al tener contacto con algún factor predisponente (consumo de determinados analgésicos con capacidad de inducir rebote, trastornos psicológicos, etc) ${ }^{3}$.

\section{DEFINICIÓN Y CLASIFICACIÓN}

A lo largo de los años la CAA ha sido conocida por diversos términos, tales como cefalea de rebote por analgésicos o ergotamina, cefalea fármaco-inducida o cefalea por abuso de analgésicos.

Fue Rappoport quien inicialmente propuso el concepto de cefalea por abuso de analgésicos. Este término se refirió al empeoramiento de la cefalea de base en pacientes con cefaleas crónicas frecuentes que tomaban analgésicos regularmente. La cefalea se inicia al cabo de 3-4 horas tras el cese del analgésico, exacerbándose durante 2-4 semanas: "periodo de lavado del analgésico". Transcurrido ese tiempo, el paciente mejora ostensiblemente ${ }^{6}$.

Sin embargo, la definición nosológica de la cefaleas primarias crónicas es controvertida. En el año 1988 la International Headache Society (IHS) publicó la primera "Clasificación y Criterios Diagnósticos de las Cefaleas, las Neuralgias Craneales y el Dolor Facial". Hasta entonces no existía consenso acerca de las características clínicas de los diferentes tipos de cefaleas, por lo que esta clasificación sirvió de instrumento tanto para la práctica asistencial como para la realización de numerosos trabajos de investigación que permitieron avanzar en el conocimiento de las cefaleas. Sin embargo, fue muy criticada sobre todo en el apartado de las cefaleas crónicas y el abuso de analgésicos, ya que todo paciente con cefalea crónica, según la IHS, debía ser diagnosticado de cefalea de tensión crónica (CTC) (no existía otra posibilidad). La migraña fue excluida de la clasificación como cefalea crónica y todos los pacientes con abuso de analgésicos se clasificaron como cefalea secundaria ${ }^{12}$.

\footnotetext{
Migraña crónica (MC)

A. Cefalea (cefalea tensional y/o migraña) de $\geq 15$ días/mes al mes durante al menos tres meses

B. Ocurre en pacientes que han tenido por lo menos 5 ataques cumpliendo los criterios de migraña sin aura

C. Cefalea de $\geq 8$ días/mes durante por lo menos tres meses que cumple los criterios $\mathrm{C} 1$ y $\mathrm{C} 2$, asociándose síntomas de migraña sin aura:

1. Al menos dos de los siguientes: localización unilateral, pulsátil, intensidad moderada o grave, se agrava con la actividad física rutinaria

2. Al menos uno de los siguientes: náusea y/o vómitos, fotofobia y fonofobia

3. Responde a triptanes o ergóticos antes de que se desarrolle C1.

D. No existe abuso de medicación y no está atribuido a otros trastornos
}

Tabla 1. Criterios IHS 2006 para la Migraña Crónica (MC).

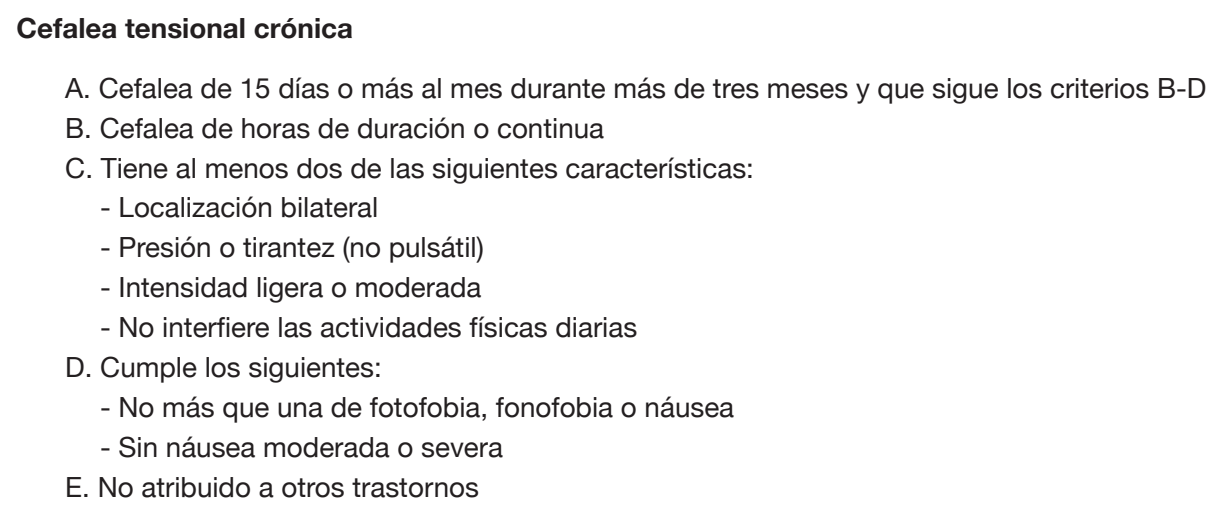

Tabla 2. Criterios IHS 2004 para la CTC. 


\section{Cefalea diaria de reciente comienzo}

A. Cefalea de más de tres meses que sigue los criterios B-D

B. Cefalea diaria e incesante desde el comienzo o menos de tres días desde el comienzo

C. Al menos dos de las siguientes características del dolor:

- Localización bilateral

- Presión/tirantez (no pulsátil)

- Intensidad leve o moderada

- No agrava la actividad física diaria

D. Cumple las dos de las siguientes:

- No más que una de fotofobia, fonofobia o ligera náusea

- No náusea moderada o intensa ni vómito

E. No atribuido a otros trastornos

Tabla 3. Criterios IHS 2004 propuestos para la cefalea diaria de reciente comienzo.

\section{Hemicránea continua}

A. Cefalea durante más de tres meses que sigue los criterios B-D

B. Sigue todas las características:

- dolor unilateral que no cambia de lado

- diario y continuo, sin periodos de no cefalea

- intensidad moderada con exacerbaciones de dolor grave

C. Al menos una de las siguientes características autonómicas ocurren durante las exacerbaciones ipsilaterales a la cefalea:

- inyección conjuntival y/o lagrimeo

- congestión nasal y/o rinorrea

- ptosis y/o miosis

D. Respuesta terapéutica completa a la indometacina

E. No atribuido a otros trastornos

Tabla 4. Clasificación IHS 2004 propuesta para la hemicránea continua.

\section{Cefalea inducida por abuso de medicación}
A. Cefalea presente $\geq 15$ días/mes que cumpla el criterio $C$.
B. Abuso regular durante $>3$ meses de uno o más medicamentos:
1. Ergotamina, triptanes, opioides o analgésicos combinados consumidos $\geq 10$ días $/ \mathrm{mes}$
2. Analgésicos simples $\geq 15$ días/mes
C. La cefalea se ha desarrollado o ha empeorado durante el abuso de medicación.

Tabla 5. Clasificación de la cefalea atribuida al abuso de sustancias o a su retirada (IHS 2006).

Los expertos establecieron la necesidad de revisar estos criterios diagnósticos, siendo la clasificación que más se aceptó la de Silberstein y colaboradores, que incluyó en la CCD a todas las cefaleas primarias que se presentan al menos quince días al mes durante un período de 6 meses y un mínimo de 4 horas al día sin tratamiento. Estos autores establecieron cuatro variedades de CCD como son: migraña transformada (MT), CTC, cefalea crónica de inicio reciente y hemicránea continua, pudiendo ir cada una de ellas con o sin abuso de analgésicos. Se excluyeron de esta definición las cefaleas crónicas secundarias, como las de causa vascular, alteraciones intracraneales, patología cervical, témporo-mandibular o postraumática. Nu- 


\begin{tabular}{lccc}
\hline Grupo & Mínima eficaz $(\mathbf{m g})$ & Recomendada (mg) & Máxima (mg) \\
Beta-bloqueantes & & & 160 \\
Propranolol & 40 & 60 & 160 \\
Nadolol & 40 & 60 & 100 \\
Atenolol & 50 & 50 & 200 \\
Metoprolol & 100 & 100 & 75 \\
Antidepresivos & 10 & 25 & 1500 \\
Amitriptilina & & 600 & 200 \\
Antiepilépticos/Neuromoduladores & 300 & 100 & 10 \\
Ac. Valproico & 50 & 5 & \\
Topiramato & & 5,5 & \\
Calcioantagonistas & & & \\
\hline
\end{tabular}

Tabla 6. Dosis diarias recomendadas de los principales tratamientos preventivos

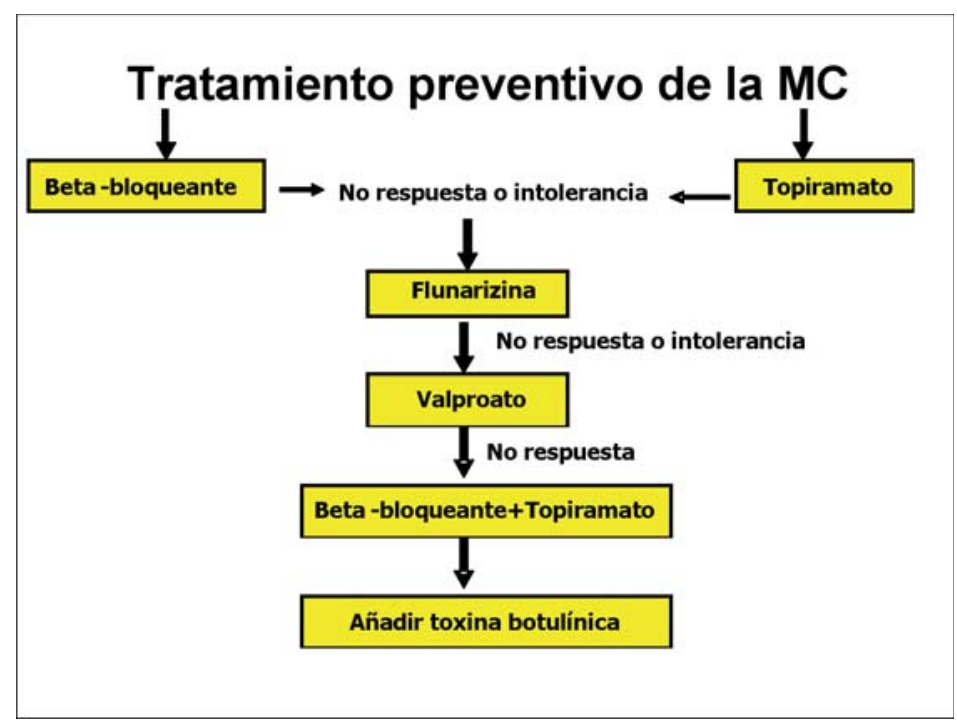

Figura 1. Tratamiento preventivo de la MC.

merosos trabajos de investigación se realizaron con esta clasificación, que sin embargo no fue aceptada por la $\mathrm{IHS}^{13}$.

Fue en el año 2004 cuando la IHS publicó la primera revisión a la clasificación del año 1988 y posteriormente una nueva revisión en el 2006, en la que reconoce los 4 subtipos de cefalea propuestos por Silberstein y colaboradores (aunque la migraña crónica -MC- no es independiente sino complicación de la migraña). Establece el abuso entre analgésicos simples, combinados, opiáceos y ergóticos. Sigue manteniendo independientes los apartados de cefaleas crónicas primarias y la cefalea por abuso de sustancias $^{14,15}$. Esta clasificación permitirá establecer unos criterios diagnósticos unificados, facilitando nuevas investigaciones y avanzando en el conocimiento de las cefaleas crónicas, aunque continúa habiendo controversia entre los autores ya que algunos creen que la CAA es una entidad primaria.

Para poder realizar un diagnóstico diferencial y un tratamiento adecuado es necesario conocer los criterios diagnósticos de los diferentes tipos de cefaleas crónicas y del abuso de analgésicos. 
La nueva clasificación de la IHS 2006 define a la MC como aquella de igual o más de 15 días de duración al mes, durante al menos 3 meses, y de estos 15 días o cumplen criterios de migraña durante igual o más de 8 días de duración al mes o responden a triptanes o ergóticos, pero siempre y cuando no exista abuso de medicación (o haber retirado durante 2 meses el abuso de analgésicos) (tabla 1)

En relación a la CTC, según la última clasificación de la IHS es la cefalea de igual o mayor duración de 15 días al mes, durante al menos 3 meses, de horas de duración o continua que cumple los criterios de cefalea tensional (tabla 2). A continuación se señalan los criterios propuestos por la IHS para la cefalea diaria de reciente comienzo y hemicránea continua aunque éstas tienen menor prevalencia que la MC y CTC (tablas 3 y 4$)$.

Los criterios de la IHS 2006 definen a la cefalea inducida por abuso de medicación como aquella cefalea presente de igual o más de 15 días/mes que se ha desarrollado o ha empeorado durante el abuso de medicación y existe abuso regular durante más de 3 meses de uno o más medicamentos usados como tratamiento agudo de la cefalea (ergotamina, triptanes, opioides o analgésicos combinados consumidos al menos 10 días/mes y/o analgésicos simples consumidos al menos 15 días/mes) (tabla 5).

\section{PERFIL CLÍNICO}

Existen un perfil clínico y una serie de signos y síntomas comunes en los pacientes con cefalea consumidores habituales de analgésicos que iremos revisando a lo largo de este apartado. La edad media de los pacientes oscila entre los 40-45 años, predomina el sexo femenino, con historia previa de cefaleas de 20-25 años de evolución y un periodo de abuso de analgésicos de 7-10 años de media.

Los pacientes con CAA aquejan una cefalea constante, diaria o casi diaria, de intensidad variable, aunque suele ser moderada, que se puede desencadenar con el más mínimo esfuerzo físico o intelectual. Se suele acompañar de irritabilidad, astenia, ansiedad, problemas con la memoria, dificultad para la concentración intelectual o depresión. Se advierte un ritmo de cefaleas que parece depender de los fármacos: el dolor comienza a primera hora de la mañana, cede parcialmente al tomar el analgésico, y vuelve a aparecer horas más tarde para mantenerse durante el resto del día. Además de esta cefalea difusa de fondo, los pacientes con antecedentes de migraña pueden presentar ataques de cefalea unilateral con síntomas gastrointestinales asociados, mientras que los pacientes con CTC no suelen discriminar entre las características de su cefalea primaria y la actual CAA.
A la cefalea crónica descrita se pueden asociar otros síntomas y signos debido al uso excesivo y continuado de los analgésicos: nefropatía, alteraciones cardiovasculares, digestivas, hematológicas, cutáneas y de anejos, junto con problemas de tolerancia, habituación y dependencia.

El uso continuado de analgésicos hace que aparezcan fenómenos de tolerancia y abstinencia. Los pacientes necesitan cada vez dosis mayores para conseguir igual efecto analgésico y experimentan cefalea de rebote cuando se suprimen los analgésicos de manera repentina.

\section{TRATAMIENTO}

El abordaje del tratamiento en los pacientes con CAA requiere una atención integral. El tratamiento debe ser médico, psicológico y físico para lograr buenos resultados.

Desde el inicio del tratamiento, el médico debe conocer los elementos de la vida del paciente que puedan influir en el desarrollo y respuesta al tratamiento de su cefalea, aspectos emocionales y psicológicos. Salvo que sean claros, éstos no deben resaltarse al inicio del tratamiento, ya que el paciente puede creer que sus quejas somáticas no son tomadas en serio.

En líneas generales el tratamiento de la CAA debe iniciarse con la información al paciente, seguido de un período de desintoxicación, dando unas pautas concretas en cuanto al tratamiento sintomático e instaurando un tratamiento preventivo.

\section{Información al paciente}

El paciente debe saber que se enfrenta a un problema crónico. Es importante explicarle en qué consiste su enfermedad y que ésta no tiene tratamiento curativo radical, pero que sí puede experimentar una gran mejoría siguiendo una serie de pautas en las que él es una parte fundamental, lo cual es indispensable para conseguir el éxito terapéutico.

Al informar sobre la CAA hay dos aspectos básicos fundamentales a transmitir al paciente: la toma habitual de analgésicos es capaz de contribuir al desarrollo de cefalea crónica y el uso de analgésicos puede impedir o reducir la acción de los tratamientos preventivos para la cefalea. Otro punto importante que debe explicarse al paciente es que el tratamiento profiláctico irá haciendo su efecto progresivamente a lo largo del tiempo y no de forma inmediata.

\section{Desintoxicación}

El abandono de los analgésicos que el paciente consume a diario es importante en el tratamiento de la CAA. Con ello puede disminuir la frecuencia de cefaleas y el tratamiento profiláctico puede ser más efectivo. Pa- 
rece bien demostrado que el abandono de ergóticos, opiáceos y analgésicos combinados es crucial para el éxito del tratamiento ${ }^{16}$, aunque los últimos trabajos realizados con topiramato como tratamiento preventivo de la MC demuestran eficacia a pesar de continuar consumiendo algún analgésico ${ }^{17,18}$.

La desintoxicación se puede realizar de forma brusca, recomendable en la mayoría de los casos, o de forma gradual y progresiva, recomendable en los casos de abuso añadido de barbitúricos, narcóticos o benzodiacepinas, para evitar síntomas de abstinencia graves.

El cese agudo del consumo de analgésicos puede originar un síndrome de abstinencia, consistente en un aumento en la intensidad de la cefalea, trastornos vegetativos (náuseas, vómitos, taquicardia, hipotensión, etc.), distimia, alteraciones del sueño, agitación, temblores, e incluso convulsiones en el caso de los barbitúricos. Estos síntomas se inician a las 24-48 horas del cese de la medicación analgésica y suelen durar entre 5-7 días. Es preferible siempre que sea posible que la desintoxicación se realice de forma ambulatoria, de este modo el paciente se implica más y pone más de su parte que en el tratamiento hospitalario.

Se recomienda tratamiento hospitalario en aquéllos que presentan: abuso de analgésicos que contengan tranquilizantes tipo codeína o barbitúricos, debido al síndrome de abstinencia que presentan los pacientes, pudiendo ser difícil el manejo ambulatorio, algún problema psicológico importante, depresión intensa, o cualquier patología médica grave que pueda complicar el proceso de desintoxicación y/o fracaso de un intento anterior de desintoxicación ambulatoria.

\section{Tratamiento sintomático}

Durante el síndrome de abstinencia es conveniente tratar los síntomas que presenten los pacientes, lo que facilitará que sigan las pautas y no abandonen el tratamiento. Para el tratamiento de la cefalea de rebote es recomendable la sustitución de los analgésicos por AINEs pautados durante 2-3 semanas. Para este fin podemos utilizar cualquiera de los AINEs recomendados por la Sociedad Española de Neurología (naproxeno sódico a dosis de $550 \mathrm{mg} / 12$ horas, dexketoprofeno $25 \mathrm{mg} / 8 \mathrm{~h}$ o ibuprofeno $600 \mathrm{mg} / 8 \mathrm{~h})^{19}$, ya que estos fármacos no parecen inducir cefalea de rebote. Como tratamiento sintomático en caso de necesidad podemos añadir triptanes. Para evitar el desarrollo de cefalea con abuso de triptanes hemos de restringir el uso de triptanes a un máximo de 2 días a la semana (2 dosis diarias). En caso necesario podemos asociar antieméticos, domperidona o metoclopramida.

\section{Tratamiento preventivo}

Como sabemos que estos pacientes, al retirar los anal- gésicos, experimentarán un empeoramiento brusco e importante de su cefalea, el tratamiento preventivo ha de ser doble, uniendo una tanda corta de AINEs con el tratamiento preventivo reglado.

A.- Tratamiento preventivo corto. Para intentar contrarrestar la cefalea de rebote, y mientras el tratamiento preventivo hace efecto, es importante pautar AINEs durante 2-4 semanas, con protección gástrica si procede. Podemos utilizar AINEs tipo naproxeno o naproxeno sódico (a dosis de $500 \mathrm{mg} / 12$ horas) o AINEs de vida media larga, tipo nabumetona (a dosis de 1 gramo/12 horas)19. En algunos pacientes rebeldes se recomienda incluso una pauta descendente de prednisona. Por último, en pacientes con patología psiquiátrica $\mathrm{o}$ en tratamiento con psicofármacos es útil la co-administración de una pauta descendente de neurolépticos, aunque en estos casos es conveniente que el tratamiento sea pautado por el neurólogo ${ }^{20,21}$.

B.- Tratamiento preventivo convencional. Aunque en pacientes con CAA algunos autores recomiendan esperar a utilizar tratamiento preventivo sólo en los casos en los que la cefalea no cede tras la desintoxicación, nosotros pensamos que en los pacientes con CAA el tratamiento preventivo está indicado desde el inicio.

En los pacientes con CTC el tratamiento preventivo de elección es la amitriptilina, a dosis bajas (20-50 $\mathrm{mg} /$ día en toma única nocturna). En el caso de la MC existen varias opciones para el tratamiento preventivo

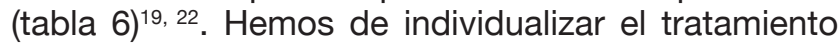
teniendo en cuenta por un lado el perfil del paciente y por otro las contraindicaciones y los efectos secundarios. Como puede observarse en la figura 1, el tratamiento preventivo de elección en estos pacientes son los beta-bloqueantes o el topiramato. En caso de no respuesta a estos fármacos, puede emplearse la flunarizina, si bien hemos de tener en cuenta que este fármaco puede inducir depresión y sobrepeso, hechos que frecuentemente impiden su utilización en este grupo de pacientes formado mayoritariamente por mujeres de edad media de la vida. La siguiente opción es el ácido valproico, fármaco con eficacia demostrada, aunque puede tener efectos secundarios serios, pues es teratógeno y su uso nos obliga al control analítico de transaminasas. Recientemente se ha demostrado que la utilización de tratamiento combinado con un beta-bloqueante y un antiepiléptico (topiramato o ácido valproico) añade un plus de eficacia probablemente por su efecto sinérgico al ser medicaciones con mecanismos de acción complementarios ${ }^{23}$. Finalmente, la infiltración pericraneal con toxina botulínica tipo $\mathrm{A}$ ha demostrado también cierta eficacia como tratamiento coadyuvante en pacientes con CAA. Este tratamiento ha de administrarse cada 3 meses y tiene la ventaja de no tener efectos secun- 
darios "centrales", por lo que es una excelente opción para pacientes con pobre tolerabilidad a los otros fármacos

\section{PRONÓSTICO}

El pronóstico real a medio-largo plazo de la CAA es poco conocido. Son escasos los estudios realizados a largo plazo en los pacientes con CAA y además los disponibles arrojan resultados contradictorios, lo que puede ser debido a diferencias metodológicas.

No está claro si existen factores pronósticos en la evolución de los pacientes con CAA. Algunos autores opinan que existen factores que influyen en el mejor o peor pronóstico. La historia previa de migraña, menor tiempo de evolución de consumo de analgésicos, el consumo de analgésicos simples y tomar tratamiento preventivo se ha relacionado con mejor pronóstico, mientras que la historia previa de cefalea tensional, mayor tiempo de evolución de consumo de analgésicos y el abuso de ergóticos, opiáceos y analgésicos combinados se han relacionado con un peor pronóstico. En lo que realmente se han puesto de acuerdo los autores es que los pacientes con CCD y consumo excesivo de analgésicos presentan peor pronóstico $24-28$.

En un reciente trabajo realizado en población general española se estudió la evolución de 72 pacientes con CAA. Un médico de familia les pautó el tratamiento (siguiendo las recomendaciones de la sociedad española de neurología) y realizó un seguimiento a los 15 días, al mes, 3 meses, 6 meses, al año y a los 4 años. Se observó que al año del inicio del tratamiento el $64 \%$ de los pacientes no cumplían criterios de abuso de analgésicos y de éstos sólo el $8 \%$ continuaba con CCD mientras que el $56 \%$ tenían cefalea episódica. A los cuatro años, el $56 \%$ de los pacientes no abusaba de analgésicos. De éstos, el $47 \%$ presentó cefalea episódica y el 9\% CCD sin abuso de analgésicos ${ }^{29,30}$.

Estos datos son muy importantes, ya que confirman la buena evolución de un gran número de pacientes con CAA tras el tratamiento, tanto a corto como a medio plazo, y en nuestra opinión justifican plenamente este tipo de intervención a nivel de Atención Primaria. Los estudios existentes en la literatura sobre la evolución y pronóstico de los pacientes con CAA presentan unos resultados positivos similares a los nuestros, aunque están realizados en unidades de cefalea y hospitales, no desde el ámbito de la Atención Primaria ${ }^{24-28}$.

Como conclusión, nos gustaría insistir sobre la importancia que tiene que el médico de familia conozca de la existencia de la CAA y su tratamiento. A lo largo de este artículo hemos visto que unos conocimientos sencillos de la enfermedad nos van a ayudar a realizar un diagnóstico y tratamiento fáciles de llevar a cabo en la mayoría de los casos desde la atención primaria. Los resultados pueden ser muy satisfactorios para los pacientes, ya que, sin tener que ser derivados al especialista, el médico de cabecera les puede ayudar a disminuir la intensidad y frecuencia de sus cefaleas, aumentando con ello su calidad de vida y disminuyendo el consumo analgésico y las patologías derivadas de éste. Con la intervención desde atención primaria también contribuimos a disminuir los costes directos de la enfermedad, como es el gasto farmacéutico, y los indirectos, que son los producidos por las patologías derivadas del consumo excesivo de analgésicos y el absentismo laboral.

\section{BIBLIOGRAFÍA}

1. Castillo J, Muñoz P, Guitera V, Pascual J. Epidemiology of chronic daily headache in the general population. Headache 1999; 39:190-6.

2. Colás R, Temprano R, Muñoz P, Castillo J, Guitera V, Pascual J. Epidemiology of chronic daily headache with analgesic overuse in a large general population simple. Cephalalgia 2001; 21:278-9.

3. Pascual J, Mateos V, Gracia M, Láinez JM. Medication overuse headache in Spain. Cephalalgia. 2008; 28(11):1234-6.

4. Lippman CW. Characteristic headache resulting for prolonged use of ergot derivates. J Nerv Ment Dis 1955; 121:270-3.

5. Isler $\mathrm{H}$. Migraine treatment as cause of chronic migraine. En: Advances in Migraine Research and Therapy. Rose Fc ed. New York: Raven Press; 1982. p. 159-64.

6. Rappoport A, Weeks R, Sheftell F. Analgesic rebound headache: theoretical and practical implications. Cephalalgia 1985; 5 (Suppl 3):448-9.

7. Mathew Nt, Reuveni U. Drug-induced refractory headache. Cephalalgia 1987; 7 (Suppl 6):154-6.

8. Pascual J. Worsening of transformed migraine with naratriptan given as a prophylactic treatment. Cephalalgia 2000; 40:399400.

9. Limmroth V, Katsarava Z, Fritsche G, Diener HC. Headache after frequent use of serotonin agonists zolmitriptan and naratriptan. Lancet 1999; 353:78.

10. Bowdler I, Kilian J, Gänsslen-Blumberg S. The association between analgesic abuse and headache: coincidental or causal? Headache 1988; 28:494.

11. Lance E, Parkes C, Wilkinson M. Does analgesic abuse cause headaches de novo? Headache 1988; 28:61-2.

12. Headache Classification Committee of the International Headache Society. Classification and diagnostic criteria for headache disorders, cranial neuralgias and facial pain. Cephalalgia 1988; 8 (supl 7):1-96.

13. Silberstein SD, Lipton RB, Solomon S, Mathew NT. Classification of daily and near-daily headaches: proposed revisions to the IHS criteria. Headache 1994; 34:1-7.

14. Headache Classification Committee of the International Headache Society. The International Classification of Headache Disorders: 2nd edition. Cephalalgia 2004; 24 (Suppl 1):9-160.

15. Headache Classification Committee of the International Headache Society. New appendix criteria open for a broader concept of chronic migraine. Cephalalgia 2006; 26:742-6. 
16. Colás R, Muñoz P, Temprano R, Gómez C, Pascual J. Chronic daily headache with analgesic overuse: epidemiology and impact on quality of life. Neurology 2004; 62:1338-42.

17. Diener HC, Bussone G, Van Oene JC, Lahaye M, Schwalen S, Goadsby PJ; TOPMAT-MIG-201 (TOP-CHROME) Study Group. Topiramate reduces headache days in chronic migraine: a randomized, double-blind, placebo-controlled study. Cephalalgia 2007; 8:962.

18. Silberstein SD, Lipton RB, Dodick DW, Freitag FG, Ramadan N, Mathew N, et al. Topiramate Chronic Migraine Study Group. Efficacy and safety of topiramate for the treatment of chronic migraine: a randomized, double-blind, placebo-controlled trial. Headache 2007; 47:170-80.

19. Grupo de Estudio de Cefaleas de la Sociedad Española de Neurología. Guías para el diagnóstico y tratamiento de las cefaleas, Mateos V, editor. Barcelona: Prous Science; 2006.

20. Silberstein SD, Peres MF, Hopkins MM, Shechter AL, Young WB, Rozen TD. Olanzapine in the treatment of refractory migraine and chronic daily headache. Headache 2002; 42:515-8.

21. Cahill CM, Hardiman O, Murphy KC. Treatment of refractory chronic daily headache with the atypical antipsychotic ziprasidone--a case series. Cephalalgia 2005; 25:822-6.

22. Silberstein S, Tfelt-Hansen P, Dodick DW, Limmroth V, Lipton RB, Pascual J, Wang SJ; Task Force of the International Headache Society Clinical Trials Subcommittee. Guidelines for controlled trials of prophylactic treatment of chronic migraine in adults. Cephalalgia 2008; 28(5):484-95.

23. Pascual J, Rivas MT, Leira R. Testing the combination betablocker plus topiramate in refractory migraine. Acta Neurol Scand 2007; 115:81-3.
24. Fritsche G, Eberl A, Katsarava Z, Limmroth V, Diener HC. Druginduced headache: long-term follow-up of withdrawal therapy and persistence of drug misuse. Eur Neurol 2001; 45:229-35.

25. Pini LA, Cicero AFG, Sandrini M. Long-term follow-up of patients treated for chronic headache with analgesic overuse. Cephalalgia 2001; 9:878-83.

26. Bigal ME, Rapoport AM, Sheftell FD, Tepper SJ. Long-term follow-up of patients treated for chronic daily headache with analgesic overuse. Cephalalgia 2002; 22:327-8.

27. Sheftell FD, Tepper SJ, Rapoport AM. Analgesic use: a predictor of chronic pain in medication overuse headache: the HeadHUNT study. Neurology 2004; 62:677.

28. Katsarava Z, Muessig M, Dzagnidze A, Fritsche G, Diener HC, Limmroth $V$. Medication overuse headache: rates and predictors for relapse in a 4-year prospective study. Cephalalgia 2005; 25:12-5.

29. Colás R, Temprano R, Gómez C, Muñoz P, Pascual J. Patrón de abuso de analgésicos en la cefalea crónica diaria: un estudio en población general. Rev Clin Esp 2005; 205:583-7.

30. Fontanillas N. Evolución de los pacientes con cefalea crónica diaria con abuso de analgésicos en población general. Tesis doctoral. Universidad de Cantabria; 2008.

\section{Otras lecturas recomendadas:}

- Goadsby PJ, Silberstein SD, Dodick DW. Chronic Daily Headache for clinicians. London: BC Decker Inc; 2005.

- Olesen J, Goadsby PJ, Ramadan N, Tfelt-Hansen P, Welch KMS. The Headaches, 3rd ed. Philadelphia: Lippincot Williams \& Wilkins; 2006. 